\title{
Evidence for the involvement of ActA in maturation of the Listeria monocytogenes phagosome
}

\author{
Cell Research (2010) 20:109-112. doi: 10.1038/cr.2009.142; published online 22 December 2009
}

\section{Dear Editor,}

Listeria monocytogenes (Lm), a Gram-positive facultative bacterium, is the causative agent of listeriosis, a food-borne disease affecting humans [1]. During infections, Lm enters phagosomes from which it escapes into the cytoplasm. Listeriolysin O (LLO) is essential, and two phospholipases play a role in this process [1]. Actin polymerization provides the propulsive force that moves the bacteria through the cytoplasm and into adjacent cells. It is initiated by the interaction of the N-terminal domain of ActA, a 639 amino acid membrane protein, with the host Arp2/3 complex. The first 156 amino acids of the $\mathrm{NH}_{2}$-terminal domain contain three regions that contribute to actin nucleation: a stretch of acidic residues ('A' region, amino acids 32-45), a cofilin homology sequence (' $\mathrm{C}$ ' region, amino acids 145-156) and an actin monomer-binding region ('AB' region, amino acids 59-102) [2].

Many studies have dissected the function of ActA in actin polymerization. In the present study, we have explored a novel role of ActA. By using ActA mutants, we provide evidence for its involvement in phagosome disruption. These studies have implicated the acidic stretch of ActA in promoting bacterial escape from the phagosome.

During a survey of the effects of mutations of several proteins of the Lm PrfA regulon [3], we found, surprisingly, that a deletion mutant in ActA was defective in permeabilizing the vacuole to the small fluorescent dye, 8-hydroxypyrene-1,3,6-trisulfonic acid (HPTS). We tested a series of ActA deletion mutants and found that all were defective in permeabilization to HPTS (Figure 1A and Supplementary information, Figure S1), which precedes escape from the phagosome [4]. An LLO deletion exhibited no permeabilization as expected (data not shown). Since ActA mutation could conceivably affect either the production of LLO or its activity, which would affect permeabilization of the vacuole, we performed hemolysis assays (Supplementary information, Data S1), and found that hemolytic activity in culture supernatants was normal for all mutant strains listed in Figure 1D (data not shown). All ActA mutants tested were sufficiently internalized to produce adequate numbers of bacteria for the assays described.

We then asked whether ActA mutants were deficient in permeabilizing the phagosome to larger molecules. We used a method in which bacteria either in disrupted phagosomes or in the cytosol bind a Lm phage cell wall binding domain protein fragment fused to YFP (CBD-YFP) [5]. We studied several mutants previously tested in the HPTS permeabilization assay. Deletions of the proline-rich (DP-L2374), actin-binding (DP-L3994) or cofilin homology (DP-L3992) domains did not significantly reduce the percentage of bacteria binding CBD-YFP(Figure 1B). However, deletion of the acidic stretch (DP-L3996) produced a significant reduction of the binding of the YFP-labeled protein (Figure 1B, Supplementary information, Figure S2). We then tested mutant strains in which charged amino acids in the acidic stretch were replaced with alanine [6]. Charged residue to alanine mutations in the $\mathrm{N}$-terminal portion of the acidic stretch led to reduction in binding of CBD-YFP (DP-L4101), but mutations in the further C-terminal region portion (DP-L4102) did not (Figure 1B). Charged residue to alanine mutation toward the C-terminal (DP-L4103 and DP-L4110) also had no effect on binding of CBD-YFP (data not shown). This narrows the portion of ActA responsible for facilitating more extensive disruption of the phagosome to less than 15 amino acids. A mutant deleted for LLO was found to produce no CBD-YFP signal (data not shown). As an additional control, all relevant strains were tested for their ability to bind CBD-YFP and all were positive (data not shown).

These findings indicated that ActA could be involved in physical escape from the phagosome. A commonly accepted method to determine entry into the cytosol is the staining of polymerized actin with fluorescent phalloidin and measurement of its colocalization with bacteria, which cannot be used with some ActA mutants. Therefore, we studied selected ActA-deficient strains by electron microscopy. As shown in Figure 1C, escape can be detected by observing close contact of bacteria with the cytoplasm. On infection with the wild-type strain, 47 of $156(30 \%)$ 
bacteria appeared to be in the cytosol at $90 \mathrm{~min}$, but for an LLO-mutant strain, the number was 1 of 296 bacteria (data not shown). With ActA-deficient DP-L3078 ( $\Delta 7-633)$, the number of bacteria in the cytosol was 6 of $102(5.9 \%)$. For a mutant lacking the acidic stretch of ActA (DP-L3996, $\Delta 31-58)$, the number was 20 of $159(12.6 \%)$. Electron microscopy with wild-type, LLO minus and DP-L3078 strains was done twice with similar results.

Several lines of evidence support a role for ActA in vacuolar disruption, including defective permeabilization of the vacuole to small and large molecules and electron microscopic studies that show fewer ActA mutants in the cytosol.
A

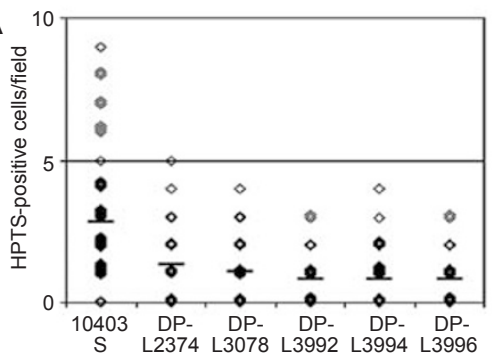

B

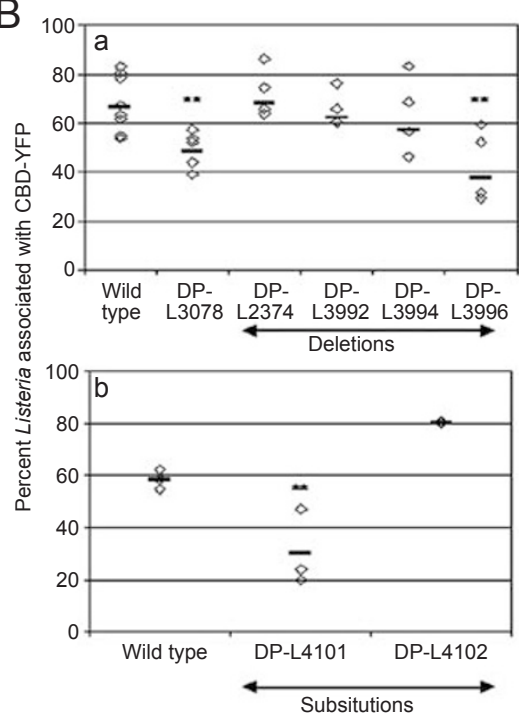

C
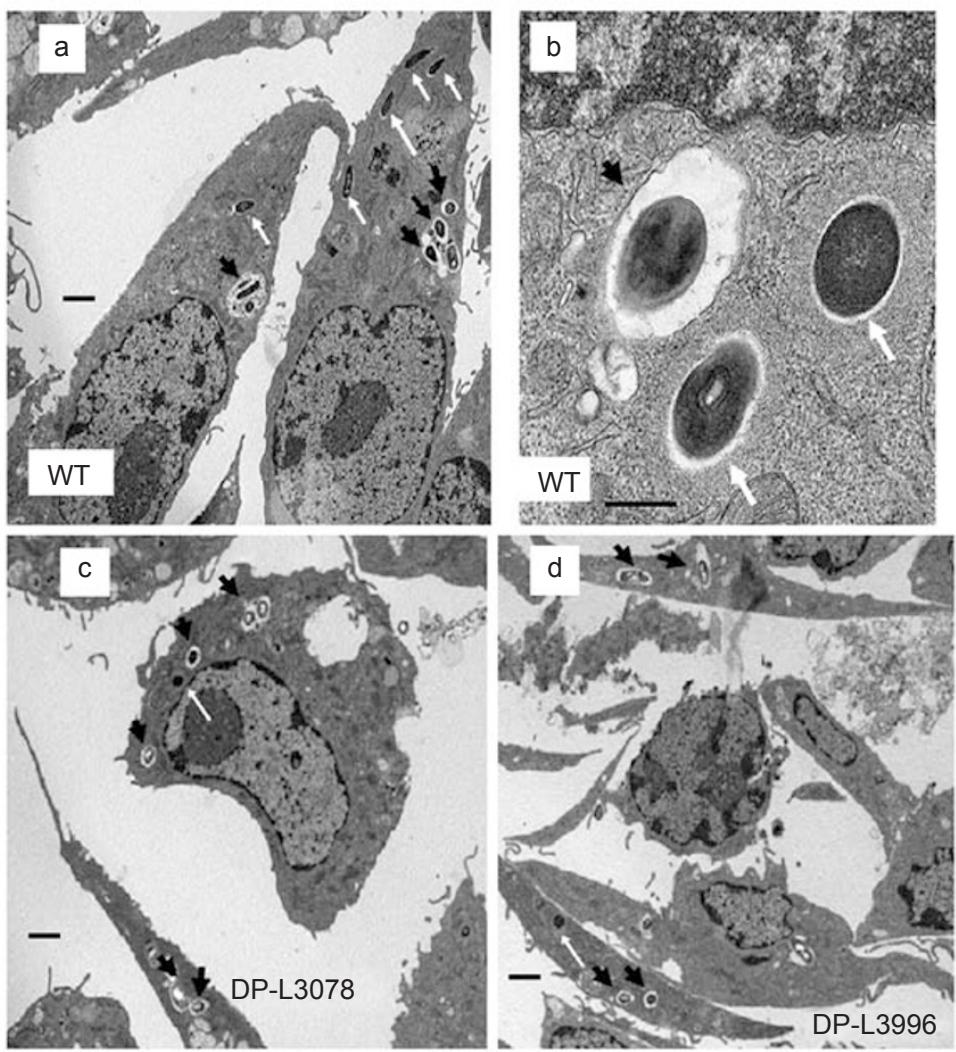

$\mathrm{D}$

Strains of $L$. monocytogenes used in this study

\begin{tabular}{|c|c|c|}
\hline Strain designation & act $A$ allele & References \\
\hline $10403 S$ & Wild type & [13] \\
\hline DP-L2161 & $\Delta h l y$ & [14] \\
\hline DP-L2374 & $\Delta 263-390$ & [15] \\
\hline DP-L3078 & $\Delta 7-633$ & {$[10]$} \\
\hline DP-L3990 & $\Delta 136-165$ & [10] \\
\hline DP-L3992 & $\Delta 146-150$ & {$[10]$} \\
\hline DP-L3994 & $\Delta 60-101$ & [10] \\
\hline DP-L3996 & $\Delta 31-58$ & [10] \\
\hline DP-L4101 & 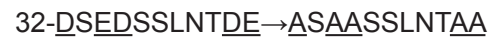 & {$[6]$} \\
\hline DP-L4102 & 44-EEEKTEEQPSE $\rightarrow \underline{\text { AAAATAAQPSA }}$ & [6] \\
\hline DP-L4103 & 60-R $\underline{\text { EETARE}} \rightarrow \underline{\mathrm{AY}} \underline{\mathrm{ATA}} \underline{\underline{A A}}$ & [6] \\
\hline DP-L4110 & 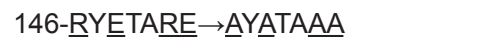 & [6] \\
\hline
\end{tabular}


Deletion of most of ActA or the acidic region (amino acids 31-58) resulted in decreased phagosome disruption, as measured by the ability of CBD-YFP to colocalize with bacteria in the infected cells. This assay probes for sufficient disruption of the phagosome to permit interactions of the bacteria with a macromolecule and has been used as a surrogate for escape. The association of CBD-YFP occurs while the bacteria are still within perforated vacuoles, which colocalize with late endosomal markers. After $\sim 25$ min of infection, bacteria were no longer associated with these markers [7].

Activity of ActA in the phagosome suggests that it is expressed prior to escape. The expression of ActA and $\mathrm{PlcB}$ (PC-PLC) is upregulated when Lm enters the cytosol [1]. However, there is evidence for activity of PC-PLC in the phagosomes of HeLa, Henle 407, Ptk2 and other cells (Supplementary information, Data S1). The expression of both LLO and PI-PLC is upregulated in the phagosome [1], yet their activities can be observed before the bacteria have entered host cells [8]. It is not unreasonable, therefore, to expect that ActA may also be expressed sufficiently to exert effects within the phagosome. A role for ActA in epithelial cell invasion by $\mathrm{Lm}$ has been reported [9].

An 11 amino acid stretch of the N-terminus of the acidic region (32-42) was shown to be important for disruption of the phagosome. This mutation has no effect on the efficacy of ActA-induced actin polymerization, but does appear to influence the stability of the protein itself [6]. Although this alanine substitution mutant is less stable than the wild-type protein, there is no evidence that the $\Delta 31-58$ protein (DPL3996), which also negatively affected disruption of the phagosome in the CBD-YFP binding assay (Figure 1B) and decreased escape from the phagosome, as seen by electron microscopy (Figure 1C), is less stable than the wild-type. ActA $\Delta 31-58$ is able to accelerate nucleation when added to the Arp $2 / 3$ complex in vitro, but was less active than full-length ActA [10].

How might ActA exert these effects? Phagosomes are surrounded by polymerized actin during and for some time after bacterial entry. Actin repeatedly assembles and disassembles close to the phagosome in epithelial cells [11]. Thus actin monomers near the phagosome membrane may enter through LLO-mediated pores, begin to polymerize and the growing chains could physically stabilize or lead to widening of the pores.

The need for the acidic region of ActA for escape suggests the existence of another mechanism that involves protein-protein interaction. The $(\mathrm{A})$ and $(\mathrm{C})$ regions of ActA interact with the host Arp2/3 [12]. It is possible that there are critical interactions with other cellular proteins that promote escape. Lastly, the actin cytoskeleton is indirectly involved with recycling endosomes, which are part of the apparatus needed for maturation of phagosomes (For further information on these interactions see Supplementary information, Data S1). There is considerable evidence that maturation is delayed by disturbing endosomal trafficking

Figure 1 (A) Effect of ActA mutation on vacuolar permeabilization. Vacuolar permeabilization was measured as previously described [3] with minor adaptations. Methods for J774 cell culture and infection have been described earlier [8]. The HPTS spectrum is $\mathrm{pH}$-dependent and the absorption, and therefore emission, wavelengths change depending on the acidity of the endocytic compartment. With an excitation wavelength of $405 \mathrm{~nm}$ there is fluorescence of the acidic compartment, which is retained when the $\mathrm{pH}$ increases, but at higher $\mathrm{pH}$, there is also fluorescence after excitation at $440 \mathrm{~nm}$. This $440 \mathrm{~nm}$-excited fluorescence appears first in permeabilized vacuoles and then in the cytosol. The number of whole cells per field acquiring fluorescence at excitation at $440 \mathrm{~nm}$ was visualized at $\times 100$ magnification, and the number of cells per field was counted for 20-30 fields per condition and per experiment [3]. Each point indicates the value for one field, — indicates the mean value for the condition. Some points overlap and are not seen in the figure. The difference between the wild-type and all mutants was significant, $P<0.05$, as determined by using the two-tailed $t$-test. (B) Effect of ActA mutation on disruption of the vacuole. RAW 264.7 cells expressing CBD-YFP were infected with either wild-type or ActA mutant strains resuspended in PBS. After $30 \mathrm{~min}$ at $37^{\circ} \mathrm{C}$, infected cells were washed with warm PBS and the culture medium was replaced with fresh medium containing $50 \mu \mathrm{g} / \mathrm{ml}$ gentamicin. At $90 \mathrm{~min}$, cells were washed with warm PBS, fixed with $4 \%$ paraformaldehyde, permeabilized with $0.1 \%$ Triton X-100 and labeled with Alexa568-phalloidin to detect polymerized actin associated with bacteria in the cytosol. The number of bacteria associated with CBD-YFP was compared to the total number of bacteria detected by DNA staining. Results are indicated as percentage of L. monocytogenes associated with CBD-YFP; bars indicate means for each set of data. Upper graph, results obtained with wild-type $L$. monocytogenes or strains deficient for all or a portion of the ActA protein. Lower graph, effect of charged to alanine substitutions in ActA. These experiments were done thrice and the results of a representative experiment are shown. ** Indicates $P<0.01$ compared to the wild-type. (C) Detection of escape by electron microscopy. J774 cells were infected as described in [3, 8] and fixed after 90 min with a solution of formaldehyde in $0.1 \mathrm{M}$ cacodylate. (b) Bacteria contained within the phagosome are seen to be in spacious vacuoles, whereas bacteria that have escaped are in close contact with the cytosol. In lower power views, the same distinction can be made (a, c, d). a and b, wildtype; c, DP-L3078 ActA $\Delta 7-633$; d, DP-L3996 ActA $\Delta 31-58$. Black arrowheads indicate some of the bacteria contained within vacuoles; white arrows indicate some of the bacteria in the cytosol. For a, c and d, the scale bars indicate 2 microns and for b, the scale bar indicates $500 \mathrm{~nm}$. 
[7]. Perhaps, actin polymerization near or within the phagosome interferes with maturation of the phagosome. Even if the effects are modest, any disruption of the processes leading to bacterial killing can result in more successful infections.

\section{Acknowledgments}

We are grateful to Dan Portnoy (University of California, Berkeley) for donation of all actA mutants. We wish to acknowledge the excellent technical assistance of Ay Jy Phoun. We thank Hélène Marquis (Cornell University) and Justin Skoble (Anza Therapeutics Inc.) for insightful comments on this manuscript. This research was supported by grants from the National Institutes of Health, NIAID, AI-45153 and from the University of Pennsylvania Research Foundation.

\section{Mathilde A Poussin ${ }^{1,2}$, Howard Goldfine ${ }^{1}$}

${ }^{I}$ Department of Microbiology, University of Pennsylvania School of Medicine, Philadelphia, PA 19104-6076, USA

Correspondence: Howard Goldfine

Tel: +1 6106647153

E-mail: goldfinh@mail.med.upenn.edu

${ }^{2}$ Current address: Ovarian Cancer Research Center-421 Curie Boulevard, Room 1326-1328A BRB II/III, University of Pennsylvania School of Medicine, Philadelphia, PA 19104, USA.

Tel: +1 2155734787

E-mail:mpoussin@mail.med.upenn.edu

\section{References}

1 Vázquez-Boland JA, Kuhn M, Berche P, et al. Listeria pathogenesis and molecular virulence determinants. Clin Microbiol Rev 2001; 14:584-640.

2 Welch MD. Actin-based motility and cell-to-cell spread of Listeria monocytogenes. In: Goldfine H, Shen H, eds. Listeria monocytogenes: Pathogenesis and host response. New York: Springer, 2007:197-223.

3 Poussin MA, Goldfine H. Involvement of Listeria monocytogenes phosphatidylinositol-specific phospholipase $\mathrm{C}$ and host protein kinase $\mathrm{C}$ in permeabilization of the macrophage phagosome. Infect Immun 2005; 73:4410-4413.

4 Shaughnessy LM, Hoppe AD, Christensen KA, Swanson JA.
Membrane perforations inhibit lysosome fusion by altering $\mathrm{pH}$ and calcium in Listeria monocytogenes vacuoles. Cell Microbiol 2006; 8:781-792.

5 Loessner MJ, Kramer K, Ebel F, Scherer S. C-terminal domains of Listeria monocytogenes bacteriophage murein hydrolases determine specific recognition and high-affinity binding to bacterial cell wall carbohydrates. Mol Microbiol 2002; 44:335-349.

6 Lauer P, Theriot JA, Skoble J, Welch MD, Portnoy DA. Systematic mutational analysis of the amino-terminal domain of the Listeria monocytogenes ActA protein reveals novel functions in actin-based motility. Mol Microbiol 2001; 42:1163-1177.

7 Henry R, Shaughnessy L, Loessner MJ, et al. Cytolysin-dependent delay of vacuole maturation in macrophages infected with Listeria monocytogenes. Cell Microbiol 2006; 8:107-119.

8 Wadsworth SJ, Goldfine H. Listeria monocytogenes phospholipase C-dependent calcium signaling modulates bacterial entry into J774 macrophage-like cells. Infect Immun 1999; 67:17701778 .

9 Suarez M, Gonzalez-Zorn B, Vega Y, Chico-Calero I, VazquezBoland JA. A role for ActA in epithelial cell invasion by Listeria monocytogenes. Cell Microbiol 2001; 3:853-864.

10 Skoble J, Portnoy DA, Welch MD. Three regions within ActA promote Arp2/3 complex-mediated actin nucleation and Listeria monocytogenes motility. J Cell Biol 2000; 150:527-537.

11 Yam PT, Theriot J. Repeated cycles of rapid actin assembly and disassembly on epithelial cell phagosomes. Mol Biol Cell 2004; 15:5647-5658.

12 Welch MD, DePace AH, Verma S, Iwamatsu A, Mitchison TJ. The human Arp2/3 complex is composed of evolutionarily conserved subunits and is localized to cellular regions of dynamic actin filament assembly. J Cell Biol 1997; 138:375-384.

13 Bishop DK, Hinrichs DJ. Adoptive transfer of immunity to Listeria monocytogenes: the influence of in vitro stimulation on lymphocyte subset requirements. J Immunol 1987; 139:20052009.

14 Jones S, Portnoy DA. Characterization of Listeria monocytogenes pathogenesis in a strain expressing perfringolysin $\mathrm{O}$ in place of listeriolysin O. Infect Immun 1994; 62:5608-5613.

15 Smith GA, Theriot JA, Portnoy DA. The tandem repeat domain in the Listeria monocytogenes ActA protein controls the rate of actin-based motility, the percentage of moving bacteria, and the localization of vasodilator-stimulated phosphoprotein and profilin. J Cell Biol 1996; 135:647-660.

(Supplementary information is linked to the online version of the paper on the Cell Research website.) 